Health \& Medicine | Kazushige Yokoyama \& Akane Ichik

\section{What can peptide-coated nanoparticles tell us about the causes of dementia?}

Kazushige Yokoyama,
Professor of Chemistry at the
State University of New York
Geneseo College, investigates
how peptides interact.
Along with Akane Ichiki, an
undergraduate student at the
institution, his team have been
investigating the peptides
involved in fibrillogenesis,
the process which occurs in
the brain to form aggregates
responsible for some of
the symptoms involved in
neurodegenerative diseases
like Alzheimer's and Parkinson's
disease. Their system involves
coating gold nanoparticles
with peptides and using
spectroscopic techniques to
investigate how these peptides
interact with the nanoparticles
and with each other.

ccording to the World Health form reversibly and are short-lived. Organization, about 50 million Some believe that this oligomer is

people worldwide are living with neurotoxic but the resulting fibrils are
not. As a result, it is difficult to identify dementia. Despite its association with a a normal part of ageing. Alzheimer's major cause of disability and dependent among older people worldwide, resulting in the progressive deterioration of cognition and movement The effect of these can be overwhelming - and not just for those with dementia, but also their carers and families.

The mechanisms which cause Alzheimer's and Parkinson's disease are poorly understood. A common thread between both diseases is that they involve the build-up or 'aggregation' of peptides in brain cells. The resulting structures - known as plaques in Alzheimer's and Lewy bodies when the neuron degeneration in both diseases.

\section{AGgREgate FORMATION}

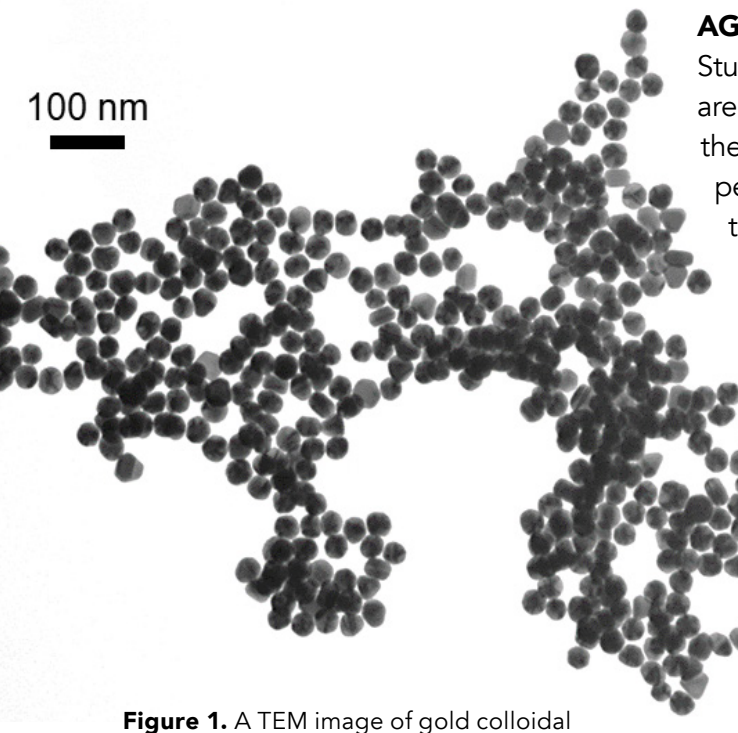

dying the way these aggregates

themed is difficult. The aggregates

eptides. The peptides combine

o form oligomers - a repeating

peptides. These continue to grow together and eventually

form fibrils, which are

major component in the toxic aggregates associated with th The process of fibril formation,
known as fibrillogenesis, is key to understanding these dise Figure 1. A TEM image of gold colloidal
particles coated with peptides in acidic However it is also difficult to investigate: the 'pre-fibril' oligomers crucial aspects of how fibrillogenesis ccurs - including how proteins eve egin to form fibrils at all. There has nn no direct investigation to identify he section of peptides which are with others in the fibril.

A SYSTEM TO CAPTURE INITIAL FIBRILLOGENESIS Yokoyama, Professor of Chemistry at the State University of New York Geneseo College, leads a group initial stage of fibrillogenesis using a system involving peptide-coated gold nanoparticles. This research has also involved the work of Akane Ichiki, an undergraduate student at the organisation. Within the project, she has co-authored a book used to investigate peptide networking

The group's system for studying the etworking between proteins involves coating gold nanoparticles with certain peptides known to form fibrils im is to capture the diseases. Their existing only at the initial stage of the fibrillogenesis by utilising the surface potential of the gold nano-particles.

Their system is particularly effective for studying the process because when the peptide-coated gold nanoparticles collect together in a network, the change, allowing the process to be quantitatively investigated using change, allowing the a drastic colour

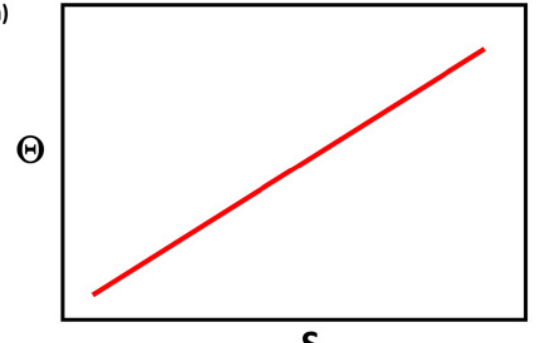

$S_{d}$

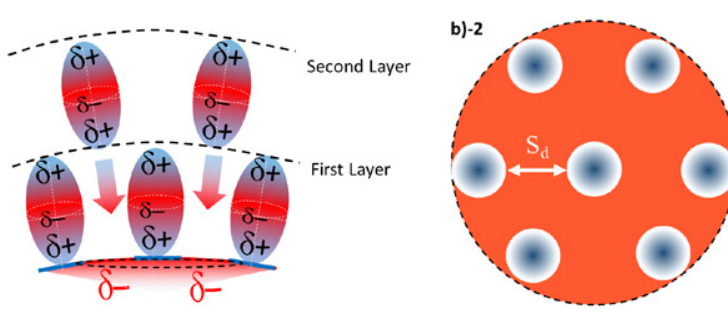

Figure 2. (a) A graph explaining the positive correlation between
and $S_{\text {. }}($ (b)-1). A side view of a prolate top peptide. (b)-2) The birds-

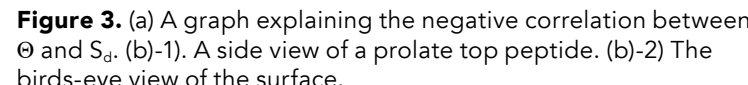

LYING DOWN OR SPIKING OUT? To understand which segment of within the fibril network, Yokoyama's group first determined how the peptides attach to the spherical surface of the gold nanoparticle.

The group investigated three types of peptides - amyloid beta 1-40 (A $\mathrm{A} 1_{1-40}$ $(\beta 2 \mathrm{~m})$ - all of which

are key components

of fibrils. The shape

of these peptides

is approximately

prolate spheroid

described as being

described as being

sphere shaped from an oval rather than

a circle. Think of a rugby ball!

The peptides therefore have two options when attaching to the nanoparticle: either in a 'lie-dow orientation, or a 'spiking-out' rientation. In outreach sessions, thama uses the example of eggs on their side or on one of their ends?

nitially, the group expected the 'liedown orientation to be favoured, with the long edge of the peptides touching the nanoparlicle. This would maximise the contact area, allowing peptides and nanoparticle.
However, the number of peptides noparticle would be imposible to explain using the lie-down orientation: each peptide would leave too much space on the surface uncovered. Based on their observed data and using a mathematical simulation, the only explanation for the calculated number of contact points was that the peptides

Using transmission electron

microscopy (TEM), the team gathered mages to investigate these collections of gold nanoparticles held together by peptide networks. Each joint conformed by peptide networking in the aggregated gold particles acts as n outreach sessions, Yokoyama uses the for the early, difficult example of eggs surrounding a beach of fibrillogenesis. peptide which end peptide which end
of the spheroid was the sphid was densely aligned small contacting areas of a sphere with 'spiking-out' peptides. This information about the peptides' orientations also allowed the team to propose which specific sections of each of the peptides might act as contact the gold nanoparticle.

NANOPARTICLES ASSEMBL The team then investigated how the peptide-coated gold nanoparticles interacted with each other. Under acidic conditions, the peptide changes stretched, and the peptidne nanoparticles assemble into collection nanoparticles, the opposite end was ting responsible for

onding to other peptides.

HOW DO PEPTIDES GLUE NANOPARTICLES TO EACH OTHER? The TEM images were used to between nanoparticles. This spectroscopic data was analysed using mathematical procedures to intere the chemistry, leading to an inter confe of how proteins change their by gold particles.

The team found the average distance between adjacent gold nano-particles 


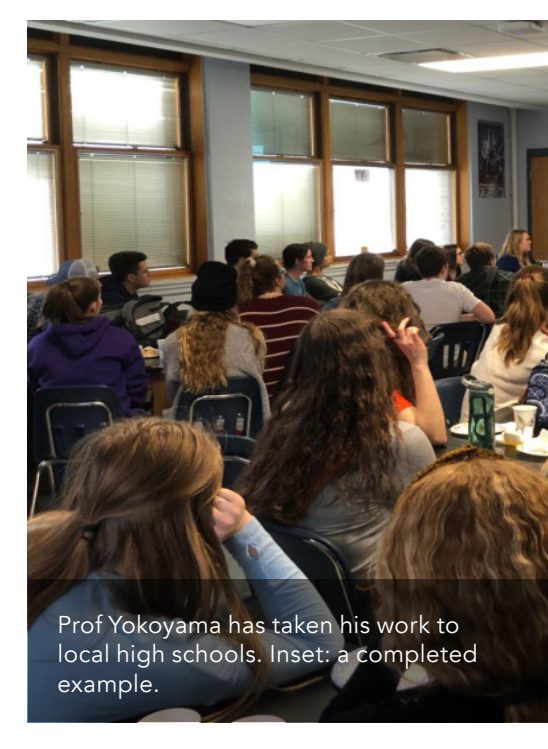

was much shorter than the length of one peptide, revealing that each the peptides around it The tangling is thought to be a result of attractions and repulsions between charges on the peptides.

REVEALING DETAIL CHARGE DISTRIBUTION OVER A PEPTIDE SURFACE

The team proceeded to reveal more chemical information for each type of peptide, by finding a general rule governing the distance between adjacent peptides $\left(S_{d}\right)$ with respect to 'coverage ratios' (0) of the gold nanoparticles by the peptides.

These experiments provide empirical evidence of the mechanisms which cause toxic aggregations of peptides in the brain in neurodegenerative diseases.

Nanoparticles coated in $A \beta_{1-40}$ or $\alpha$-syn achieved denser packing by strongly inviting a second layer of peptides to form as the distance between adjacent peptides of the first layer increased. It was speculated that these peptides have a slightly positive charge on their top and bottom ends or 'spikes', as we as a slightly negative charge around their middles. These partial charges are a result of the specific amino acid sequences in the peptides.

It helps to imagine that a surface of gold nanoparticles is like a sea of (sea of electrons). When the distance between each 'spike ship" is greater, there appears to be more space to place 'spike ships'. These extra 'spike ships' correspond to a secondary approached to network, resulting in greater density.

However, the opposite trend is seen distances between each peptide actually lead to a lower density of $\beta 2 \mathrm{~m}$ peptides. This is because $\beta 2 \mathrm{~m}$ for $\beta 2 \mathrm{~m}$-coated nanoparticles - greater relatively small region of its spikes has the regin possesses a wit most of Unlike the other two peptides, this peptide was found to prefer to gyra like a spinning top.

The more distance between two adjacent peptides, the more the $\beta 2 m$ gyrated, with a larger tilting angle. This creates more partial positive regions, discouraging the second layer of peptides from attaching to the nanoparticle due to the repulsion between positive and positive charges, causing a lower density.

APPLICATIONS AND IMPLICATIONS

Yokoyama's team has found evidence that there is a general principle share with different neurodegenerative diseases, when they interact with solid surfaces. All peptides tested in this work orientated themselves in a way to maximise networking with the other peptides. This is important for understanding how peptides interact during fibrillogenesis and may help us understand other diseases too.

Peptide-coated nanoparticles have important applications in biosensors, disease controlling materials and cellular control. Most crucially, these experiments provide empirical evidence of the mechanisms which cause toxic ggregations of peptides in the brain

A spiking-out orientation was proven to be highly interactive in a spike protein (s-protein) characteristic of the SARS-CoV-2 virus, responsible for the COVID-19 outbreak. As an s-protein binds to a cell receptor during cell infection, a conformational change can occur, triggering an effusion process followed by a replication of the virus. This process involves similar chemistry observed in this study where a spiking out peptide changes the conformation under acidic conditions, before

peptide-networking occurs. Notably, the approach used in this study may well be helpful for understanding how providing sppoten attacks cells, providing clues about how to prevent

\section{Behind the Research}

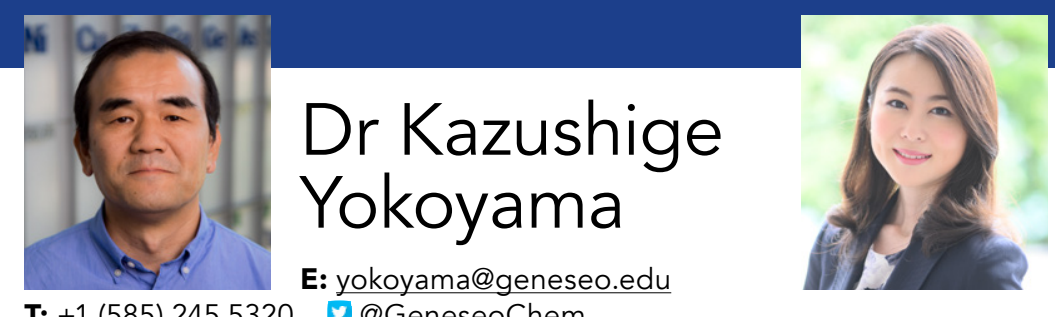

Akane

Ichiki

in https://www.linkedin.com/in/

W: $\mathrm{W}$ https://www geneseo edu/chemistry/Yokoym

Research Objectives

Prof Yokoyama uses nano-gold particles to examine what makes proteins bond. His work has potential to be applied to any proteins located over a spherical shape, including the spike proteins observed in the novel coronavirus.

\section{Detail}

Department of Chemistry

The State University of New York

Geneseo Colleg
ISC $326 \mathrm{~F}$

1 College

Geneseo, NY 14454 USA undergraduate student at the State (USA) under the Bion Geneseo College She has co-authored four publications on the nano-interface structure of amyloidogenic peptides. Ichiki is the

Kazushige Yokoyama earned his BS/MS degree from Kobe University (Japan) and PhD from the University of Colorado at Boulder (USA). He completed a postdoctoral fellow at the University of Minnesota (USA). He is currently a Professor of Chemistry Geneseo College (USA) and the co-founder of International Pe or (IPM) in Geneseo.

Funding

Gerrion Gerry Rhodes Fellowship, Kenny Lipkowitz Fellowsh Foundation, SUNY Geneseo Chemist

Department Alumni Donatio

Collaborators

essica Battaglia, Kieran Brown, Jon Deshmukh, Kaylee Hausrath, Jack Jenkins, Nichole Ralbovsky, Peter Shevlin, Leilani Helmer

Acknowledgement Mr Bob Callens (Physics Teacher) and the students of the STEM Club (NY USA) wals-Lima High School for hosting the highly acknowledged demonstration mentioned in this article.

\section{References}

Yokoyama, K., Brown, K., Shevlin, P., Jenkins, J., D'Ambrosio, E., Ralbovsky, N., Battaglia, J., Deshmukh,

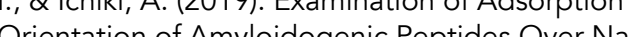

Gold Colloidal Particle Sufices Internation Nanomolecular sciences, 20(21), 5354. https://doi.org/10.3390/ ijms20215354

Yokoyama, K. and Ichiki, A. (2020). Oligomerization and Adsorption Krontation, A. (2020)idogenic Peptides Over Chemistry Research, 61, 139. Edited by James C. Taylor, Nova Science Publishers, Inc. https://novapublishers.com/ shop/advances-in-chemistry-research-volume-61/

\section{Personal Response}

How could knowledge of peptide-solid surface ther then nelp us to understand and treat diseast

II As represented by cells, skin, organs or bones, the himan body contains so many sold surfaces. Thus, observed all over the human body. A principle found in this study concluded that an energetically preferred path for a protein to interact with a solid surface is to create a maximum coverage by working together with the other proteins. With this principle, it allows us to to design the drugs which would effectively tases or particular protein causing a disease.

\section{GENESEO}

